\title{
LA FORMACIÓN DEL JUICIO EN SANTO TOMÁS DE AQUINO
}

\author{
Carmen Segura Peraita \\ Universidad de Navarra
}

Tomás de Aquino sostiene sin vacilaciones la afirmación clásica, según la cual el juicio es la sede propia de la verdad, ${ }^{1}$ definiéndola habitualmente como una comparación de conceptos. ${ }^{2}$ Esta última afirmación entraña, sin embargo, algunas dificultades que es preciso considerar, tanto de orden gnoseológico, como lógico y psicológico.

En este trabajo se intentará únicamente destacar primero, para resolver después, aquellos problemas que se plantean en el ámbito psicológico, siguiendo el pensamiento de-Tomás de Aquino.

En el momento en que nos decidimos a estudiar la formación del juicio, se plantean cuestiones como las que, a continuación -sin pretensiones de exhaustividad-se enuncian: ¿Cómo forma la inteligencia el concepto complejo que se expresa en la proposición? ¿Cómo se distinguen, en cuanto a su génesis, el concepto -fruto de la simple aprehensión- de la proposición, fruto del juicio? ¿Cómo es posible, en términos psicológicos, la comparación en que consiste la segunda operación? ¿En qué se apoya la inteligencia para componer y dividir sus conceptos? ¿En virtud de qué puede el entendimiento relacionar sujeto y predicado? ¿Puede acaso nuestra inteligencia ser informada simultáneamente por más de una especie inteligible? ¿Es posible saber de algún modo -atendiendo precisamente a la constitución de la segunda operación- si nuestras composiciones y divisiones judicativas se atienen a la naturaleza de las cosas, a lo real extramental? En definitiva, ¿estamos autorizados a afirmar que el juicio es la sede propia de la verdad?

1 «EI entendimiento no conoce la verdad sino componiendo o dividiendo por su juicio (...). Es patente que la verdad y la falsedad como en quien la conoce y dice no está sino en la composición y en la división». In I Peri hermeneias, lect. Ill, $\mathrm{n}^{\circ} 31$ (9), in fine. Y tambien: «la verdad no está en las cosas, sino en la mente, y además, en la composición y división». I $\mathrm{Vl}$ Metaphysicorum, lect. IV, $\mathrm{n}^{\circ} 1236$. Los lugares de la obra tomista en que aparece esta afirmación son muy numerosos. He citado únicamente estos dos como ejemplo, y también por la relevancia que -como se verá- tienen para la cuestión que nos ocupa.

2 Cfr.: In I Peri hermeneias, proemiun, $\mathrm{n}^{\circ} 1$ (1). 
Al intentar responder a estas cuestiones, seguiré, en la medida en que lo permita la interna conexión del discurso, el orden -no casual- en que acaban de ser formuladas. Por ello será preciso comenzar por el estudio de la formación del concepto complejo. Enseguida nos encontramos metidos de lleno en el asunto central de este trabajo: la formación del juicio. Por último, y casi como una ineludible consecuencia de lo anteriormente tratado, se analizará el modo en que la inteligencia puede conocer varias cosas mediante una sola especie.

Para abordar ya el primer asunto, se pueden tomar como punto de partida las siguientes palabras de Tomás de Aquino:

«Lo entendido se tiene como constituido o formado por la operación del entendimiento: bien sea ésta la quididad simple, bien la composición o división de la proposición. Pero para cada una de estas operaciones se entiende previamente la especie inteligible por la que el entendimiento se pone en actor. ${ }^{3}$

Dos afirmaciones igualmente relevantes para nuestro propósito se contienen en el texto citado. En primer lugar, sostiene Santo Tomás que lo entendido es constituído por la operación del entendimiento. En segundo, mantiene que, previamente, se ha de entender la especie inteligible y ello da tal forma que sin la actualización por parte de dicha especie, se hace imposible el acto de conocimiento. ${ }^{4} \mathrm{La}$ especie, es por tanto, condición sine quae non. Es preciso tener esto en cuenta ya que, el hacerlo nos permitirá enseguida abordar en toda su profundidad la cuestión central de la formación del juicio.

Ahora bien, el acto de conocimiento no se reduce -se ha apuntado ya- a la información; es algo propio; en cierto modo, exclusivo del entendimiento. Así, una vez que ha sido actualizado, está ya en condiciones de «pronunciar su verbo»; lo que también ocurre a nivel de sensibilidad, pues las facultades sensitivas han de ser igualmente informadas por su correspondiente especie, dando lugar - una vez actualizadas- a su correspondiente especie expresa.

Sin embargo, Tomás de Aquino afirma que el entendimiento puede originar tanto un concepto simple, cuanto una proposición, lo cual no es posible para los sentidos, ni tan siquiera a un nivel inferior. Habremos de preguntarnos, por tanto, qué es lo que hace posible el juicio de la inteligencia, distinguiendo netamente el conocimiento sensible del intelectual. La respuesta la proporciona nuestro autor cuando, tratando de la misma cuestión que ahora llevamos entre manos, explica que «La especie visible no se considera como lo que se ve, sino en lo que se ve. Y lo mismo respecto del entendimiento posible, salvo que el entendimiento reflexiona sobre sí mismo y sobre su especie y la vista no».5

3 De Spiritualibus Creaturis, q. un., a. 9, ad. 6. Cfr. también: De Veritate, q. 3, a. 2c.

4 Cfr.: De Potentia, q. 8, a. 1c. in medio.

5 De Spiritualibus Creaturis, q. un, a.9, ad. 6. 
Así pues, la reflexión es lo que diferencia ambos niveles de conocimiento. Tomás de Aquino no afirma todavía explícitamente que sea la reflexión lo que hace posible el juicio, que es lo que aquí trataré de mostrar. No obstante el texto es ya suficientemente significativo. ${ }^{6}$

Existen, por otra parte, otros textos en los que explícitamente sostiene Santo Tomás que el juicio es posible por la reflexión del entendimiento. Particularmente claro resulta el que reproduzco a continuación:

«El entendimiento posee en si mismo la semejanza de la cosa entendida, en cuanto que concibe las razones de los incomplejos. Sin embargo, no juzga por esta misma semejanza, sino sólo cuando compone y divide. Pues cuando el entendimiento concibe lo que es animal racional mortal, tiene en sí mismo la semejanza del hombre, mas no conoce por ello que él tiene esta semejanza, ya que no juzga que el hombre es animal racional y mortal; y por eso en esta única segunda operación del entendimiento hay verdad y falsedad, en cuanto que el entendimiento no solo posee la semejanza de la cosa entendida, sino que además reflexiona sobre la misma, conociéndola y juzgándola. Por todo esto se hace patente que la verdad no está en las cosas, sino en la mente, y además en la composición y división»?

Así pues, en cuanto el entendimiento «concibe las razones de los incomplejos» tiene ya el concepto formal, «la semejanza de la cosa», la especie expresa; ya lo hemos visto. Sin embargo, continúa diciendo Tomás de Aquino, eso no basta para el juicio, porque no conoce todavía que él tiene la semejanza. Para que dicho conocimiento se dé, es decir, para que se dé el juicio, es precisa la reflexión. Las palabras de nuestro autor no ofrecen lugar a dudas; sólo en ella reflexiona la inteligencia sobre dicha semejanza, conociéndola y juzgándola. ${ }^{8}$

Analizando el juicio desde un punto de vista psicológico, nos hallamos irrenunciablemente ante la reflexión. Si se pretendiese únicamente un tratamiento lógico e incluso psicológico, quizá bastase con considerar la composición y la división, la afirmación y la negación, como lo característico del juicio. Mas cuando se desea -por decirlo de algún modo-estudiar el juicio en su génesis, es necesario atender a la reflexión. No, desde luego para negar que consiste en una síntesis, sino más bien para llegar hasta la raíz de ese carácter sintético, descubriendo así que la composición y división se da en virtud de la reflexión, y que sólo por obra de ésta son posibles.

Cuando los comentadores tomistas de mediados del XIX y principios del XX repararon en la importancia de la reflexión dentro de la teoría tomista del juicio, las mayores divergencias interpretativas surgieron a la hora de determinar cuál era su objeto. Para unos era la especie

6 A mi juicio, tratando de exponer fielmente el pensamiento de Santo Tomás, cuando el verbum que expresa o pronuncia la inteligencia es un concepto simple, ha reflexionado ya sobre la especie inteligible y sobre su propio acto, llegando hasta el fantasma. Sin embargo, porque el tratamiento de este asunto nos alejaría demasiado del propósito (...) central, no voy a tratar ahora de esta cuestión, centrándome directamente en la reflexión judicativa.

7 In Vl Metaphysicorum, lect. IV, $\mathrm{n}^{\circ} 1236$.

8 Cfr.: Ibidem. 
inteligible; para otros, el simple incomplejo; para algunos la representación sensible; para los de más allá, el concepto complejo, etc.

Desde entonces y hasta el momento presente, las nuevas propuestas de solución y las aclaraciones o concreciones a lo ya dicho, han sido casi ininterrumpidas. La razón de esta profunda discrepancia hay que ir a buscarla, al menos en buena parte, en los propios textos de Tomás de Aquino pues, al menos a mi modo de ver. No siempre está claro lo que quiere decir, por lo que toca a la reflexión judicativa.

No obstante, puesto que no pretendo ahora hacer una exposición sistemática acerca de la reflexión, me limitaré a intentar un análisis detenido del texto contenido en los comentarios a la Metafisica aristotélica anteriormente citado.

Al leerlo atentamente parece claro que Tomás de Aquino está refiriéndose al concepto simple, fruto de la primera operación, sin embargo, no dejan de presentarse algunas dificultades que es preciso resolver. La primera y principal es consecuencia del uso analógico que de la voz «semejanza» hace Santo Tomás en distintos lugares de su obra. En efecto, no siempre la utiliza para significar lo mismo, y mientras que mediante ella designa unas veces la especie impresa, otras designa la especie expresa, o bien apunta, sin mayores precisiones, a la especie inteligible en general. Pero es preciso determinar con exactitud a qué alude cuando indica que la inteligencia reflexiona sobre la semejanza, puesto que no parece lo mismo que vuelva sobre la especie o que lo haga sobre el concepto. Así, si se considera que alude a la especie impresa, cabría pensar que la reflexión es incluso previa al acto de simple aprehensión, puesto que la forma inteligible lo precede y lo hace posible. Mas si se piensa que Tomás de Aquino está apuntado al concepto formal, de nuevo nos encontramos ante una disyuntiva, ya que -en ese caso- la reflexión podría operar tanto sobre la aprehensión simple como, incluso, sobre el juicio mismo. ${ }^{9}$

Lo cierto es que son poderosas las razones que, a pesar de las discrepancias, inducen a pensar que Tomás de Aquino se está refiriendo a una reflexión sobre la aprehensión simple. Pienso que, atendiendo al contexto y teniendo en cuenta su sentido, no se puede llegar a otra conclusión. En primer lugar porque el autor señala que la inteligencia posee la semejanza de la cosa al concebir los conceptos incomplejos, y además porque desde el principio se refiere a «la semejanza de la cosa entendida». ${ }^{10}$ Por otra parte continúa diciendo que no juzga por la semejanza misma, sino que lo hace cuando compone y divide, y está claro que lo que el entendimiento compara son sus conceptos simples. Por otra parte, los propios ejemplos de Santo Tomás conducen a esto:

«Cuando el entendimiento concibe lo que es animal racional mortal tiene en si mismo la semejanza del hombre»."11

9 Cfr.: Quodlibetum V, a. 9. y también Cfr.: Opusculum, 13: «De differentia verbi divini et humani».

10 Cfr.: In Vl Metaphysicorum, lect. IV, no 1236.

11 Cfr.: Ibidem. 
Lo que concibe es la semejanza, es decir, el verbo mental. Por último, dice que por la segunda operación el entendimiento conoce y juzga, pero también sabemos que lo que se conoce es el concepto, mientras que la especie es aquello en lo que se conoce. Así pues, lo que el entendimiento compone y divide son los conceptos obtenidos mediante la primera operación; y aquello sobre lo que reflexiona es el concepto que representa a la cosa:

«el entendimiento no sólo posee la semejanza de la cosa entendida, sino que además reflexiona sobre la misma conociéndola y jużándola». ${ }^{12}$

Ahora bien, anteriormente hemos visto que, no sólo para formar sus conceptos simples sino también para formar los compuestos, el entendimiento precisa de la especie inteligible. ${ }^{13}$ No obstante es lógico pensar que, según se trate de la primera o de la segunda operación, lo que el entendimiento logra, a partir de la especie pero por su operatividad propia, será diverso. Pues bien, lo que, precisamente en virtud de la reflexión logra la inteligencia, es la predicación. En efecto, reflexionando sobre la semejanza de la cosa llega hasta la especie y, al volver sobre ella llega a conocer nuevas perfecciones de las cosas que antes no había aprendido. Esas determinaciones, que se sitúan en la línea de la forma, son las que en el juicio se predicarán de la cosa significada por el concepto-sujeto. ${ }^{14}$ Cómo tenga esto lugar, y cómo sea posible la elaboración de varios conceptos -sujeto y predicado- lo veremos enseguida al estudiar la posibilidad o imposibilidad de que varios conceptos se hallen simultáneamente en el entendimiento. Por el momento bastará con adelantar que la unidad radical existente en la proposición se funda en la presencia de la especie inteligible ante el entendimiento, puesto que los conceptos que forman parte de la misma enunciación se forman necesariamente por la acción y desde la especie impresa que actualiza la inteligencia y, correlativamente, por la reflexión intelectual que permite llegar a conocer las restantes determinaciones de una cosa. ${ }^{15}$

Así tiene que ser ya que, de lo contrario, la inteligencia no sabría que tal predicado pertenece a tal sujeto, puesto que la unidad del objeto permanece inalterable en el nivel de la especie inteligible, pero no en el de los conceptos, que son formación intelectuales que no existen en la cosa. Es reflexionando sobre su propia operación como el entendimiento está en condiciones de considerar que algo pertenece al sujeto, en cuyo caso une componiendo ambos conceptos, o de considerar que algo no le pertenece, en cuyo caso separa mediante la división intelectual. Así es como el entendimiento compara un concepto con otro: refiriéndose a la misma especie y conociendo, por tanto, sujeto y predicado como un todo. Es entonces cuando el entendimiento conoce y dice la verdad, la adaequatio rei et intellectus. ${ }^{16}$ Por otra parte, es la «vuelta» sobre la forma inteligible lo que hace posible referir a la realidad todos nuestros

12 Cfr.: Ibidem.

13 Cfr.: De spiritualibus Creaturis, q. un, a. 9, ad. 6.

14 Cfr.: In Vl Metaphysicorum, lect. IV, $\mathrm{n}^{\circ} 1234$.

15 Cfr.: Summa Theologiae, I, q. 58, a. 2c.

16 Cfr.: In I Peri hermeneias, lect. Ill. In Vl Metaphysicorum, lect. IV. De Veritate, q. 1, a. 1c. 
conocimientos y afirmar, como hace Tomás de Aquino reiteradamente, que las concepciones del entendimiento se reducen a las cosas como a su causa.

En último extremo, por otra parte, es precisamente la posibilidad de que el entendimiento en el acto de juzgar se refiera al concepto formal y por él a la misma especie, lo que permite comprender cómo en un solo acto de conocimiento se pueden comparar simultáneamente dos conceptos.

En cierto modo ya hemos resuelto la última cuestión que nos quedaba por tratar, pues en todo el análisis precedente ha quedado suficientemente claro que la atribución es posible en virtud de la referencia a una única especie inteligible. No obstante, algo más cabe aún precisar.

Señala Tomás de Aquino que algunas cosas se pueden aprehender o bien como muchas, o bien como una, y que nuestra inteligencia puede captar a la vez el sujeto y el predicado, en cuanto que son parte de una sola proposición. ${ }^{17}$ En el texto que ahora mismo he parafraseado continúa afirmando nuestro autor que muchas cosas

«en cuanto que están unidas en un inteligible (así) se entienden simultáneamente. Mas cada cosa es inteligible en cuanto su semejanza está en el entendimiento. Luego todas aquellas cosas que se pueden conocer mediante una sola especie inteligible se conocen como un solo inteligible, y por eso se conocen simultáneamente». ${ }^{18}$

Es cierto que los conceptos del juicio se pueden aprehender también separadamente, pero lo propio es que se conozcan formando parte del mismo, pues es así como forman radicalmente algo uno, tanto si se trata de una proposición afirmativa como negativa, pues solo así se significa plenamente algo in rerum natura. ${ }^{19}$

No se entiende del mismo modo una cosa en su conjunto que en cada una de sus partes; el entendimiento no opera de la misma forma en ambos casos. Y otro tanto sucede según realice la primera o la segunda operación. Mediante la simple aprehensión, capta por separado, formando distintos conceptos simples relativos a las diferentes determinaciones de una cosa. Mediante el juicio, une las aprehensiones simples, pero de tal modo que forma un único concepto; por eso dice Santo Tomás que, a partir de la especie inteligible, se puede formar tanto un simple incomplejo como una enunciación, porque en cada caso la inteligencia «trabaja» de forma distinta. Antes entendía los conceptos como distintos; ahora los entiende como uno, lo cual es posible por la capacidad de la inteligencia de afirmar y negar, de componer y dividir. Por eso la simultaneidad judicativa constituye una verdadera unidad fundada en el objeto y en la misma operatividad intelectual. ${ }^{20}$

Quizá sea ésta la razón por la que Tomás de Aquino, y con él sus comentadores, utilizan el término «concepto» tanto para designar las aprehensiones simples como para designar las

17 Cfr.: Summa Theologiae, I, q. 58, a. 2c.

18 Ibidem.

19 In I Peri hermeneias, lect. V, no 68(17) in principio.

20 Cfr.: Joannis a Sancto Thoma, Cursus Theologius Thomisticus, ed. Leonina, Roma, 1934, disp. 22, ㄲo 26. 
complejas. Y esto mismo puede ayudar a penetrar en la profunda unidad de la proposición, que supera con mucho la mera contiguidad espacial o temporal e incluso la simple síntesis de varios elementos dispersos. Unidad que, en último extremo se funda en la composición real de las cosas, adecuadamente significada en la especie inteligible, raíz y medio de enlace de nuestros conocimientos. Unidad que a nivel judicativo se funda, en definitiva, como he apuntado, en la operatividad intelectual, pues su causa real no produciría el efecto si el entendimiento no pudiese reflexionar sobre la especie inteligible al conocer su objeto propio.

$\mathrm{Al}$ intentar resolver las cuestiones planteadas al comienzo de este trabajo, dos elementos del conocimiento intelectual se nos han hecho especial y permanentemente presentes: la especie impresa, condición de posibilidad tanto de la aprehensión simple cuanto del juicio; y la reflexión en la que, según se ha podido apreciar, consiste el juicio, la comparación y división. Dos elementos a los que ha sido preciso recurrir para tratar de resolver desde la primera dificultad hasta la última. Dos elementos que tienen -como espero que se haya podido apreciar- una singular relevancia en la formación del juicio. Ahora, tras haber pretendido sortear todos los escollos, podemos concluir sosteniendo que el entendimiento puede juzgar, es decir, componer y dividir, porque puede reflexionar sobre su acto de simple aprehensión. Al hacerlo, la inteligencia llega hasta la especie inteligible que dio origen al concepto, y puede, de este modo, conocer nuevas determinaciones, tanto esenciales como accidentales, de la cosa representada por la especie y significada por el concepto incomplejo que, una vez formada la proposición, cumplirá las veces de sujeto. A ese sujeto es al que, mediante la reflexión, el entendimiento atribuirá las nuevas determinaciones conocidas, y significadas en la proposición por el predicado. 